\title{
Clinical Factors Associated with Hepatocellular Iron Deposition in End-stage Liver Disease
}

\author{
Amelia Fierro-Fine ${ }^{1 \dagger}$, Leana Guerin ${ }^{1 \dagger}$, Hasan Hicsasmaz ${ }^{2}$ and Kyle E. Brown*3,4,5 \\ ${ }^{1}$ Department of Pathology, University of Iowa Roy J. and Lucille A. Carver College of Medicine, Iowa City, IA, USA; \\ ${ }^{2}$ ObjectRelational LLP, Iowa City, IA, USA; ${ }^{3}$ Division of Gastroenterology-Hepatology, University of Iowa Roy J. and Lucille A. \\ Carver College of Medicine, Iowa City, IA, USA; ${ }^{4}$ Program in Free Radical and Radiation Biology, University of Iowa Roy J. and \\ Lucille A. Carver College of Medicine, Iowa City, IA, USA; ${ }^{5}$ Iowa City Veterans Administration Medical Center, Iowa City, IA, USA
}

\section{Abstract}

Background and Aims: Hepatocellular iron accumulation in patients with chronic liver disease has been linked to adverse outcomes. The objective of this study was to identify clinical factors associated with hemosiderosis. Methods: A total of 103 consecutive liver transplant recipients were identified, in whom liver biopsy had been performed prior to transplantation. Laboratory and clinical data at biopsy and transplant were abstracted from the medical records and hepatocyte iron was graded in the biopsy and explant. The association of change in iron score from biopsy to transplant, with the time interval between these two events, was examined using linear mixed model analysis for repeated measures. Results: Most subjects had advanced fibrosis (F3-F4) at liver biopsy, which was performed on average about 2.5 years before transplant. Over $80 \%$ of patients had no or $1+$ hepatocyte iron at biopsy; iron increased between biopsy and transplant in about $40 \%$. The only demographic or clinical feature that correlated with increased iron was the presence of a transjugular intrahepatic portosystemic shunt. Increased iron at transplant was associated with higher serum iron and transferrin saturation at biopsy, and with lower hemoglobin level, greater mean corpuscular volume, mean corpuscular hemoglobin and mean corpuscular hemoglobin concentration, higher ferritin and model for end-stage liver disease score at transplant. Conclusions: The development of hemosiderosis in end-stage liver disease is associated with lower hemoglobin levels and alterations in red blood cell indices that are suggestive of hemolysis. These observations suggest that extravascular hemolysis may play a role in the development of secondary iron overload.

Citation of this article: Fierro-Fine A, Guerin L, Hicsasmaz $\mathrm{H}$, Brown KE. Clinical factors associated with hepatocellular

Keywords: Erythrocyte indices; End-stage liver disease; Hemolysis; Hemosiderosis; Humans; Iron.

Abbreviations: $\mathrm{HH}$, hereditary hemochromatosis; INR, international normalized ratio; $\mathrm{MCH}$, mean corpuscular hemoglobin; $\mathrm{MCHC}$, mean corpuscular hemoglobin concentration; MCV, mean corpuscular volume; MELD, model for end-stage liver disease; TIBC, total iron binding capacity; TIPS, transjugular intrahepatic portosystemic shunt.

Received: 27 March 2020; Revised: 18 May 2020; Accepted: 19 June 2020

Current addresses: St. Cloud Pathologists, P.A., St. Cloud, MN, USA (AFF); Lawrence Clinical Laboratory, Lawrence Memorial Hospital Pathology Department, Lawrence, KS, USA (LG)

*Correspondence to: Kyle E. Brown, Division of Gastroenterology-Hepatology, University of Iowa Roy J. and Lucille A. Carver College of Medicine, 4553 JCP, 200 Hawkins Drive, Iowa City, IA 52242, USA. Tel: +1-319-384-6579, Fax: +1-319356-7918, E-mail: kyle-brown@uiowa.edu iron deposition in end-stage liver disease. J Clin Transl Hepatol 2020;8(3):231-239. doi: 10.14218/JCTH.2020.00022.

\section{Introduction}

Evidence of dysregulated iron metabolism is frequently encountered in patients with chronic liver disease. This phenomenon has been studied extensively in patients with chronic viral hepatitis and nonalcoholic fatty liver disease, among whom as many as a third or more have raised serum iron levels and/or transferrin saturation. Elevated serum ferritin levels are even more common, with some studies reporting that over half of patients with these forms of chronic liver disease have hyperferritinemia. Abnormal serum iron parameters do not reliably predict increased hepatic iron content in these circumstances and hemosiderosis secondary to chronic liver disease tends to be relatively mild and infrequent in noncirrhotic livers. ${ }^{1-7}$ In contrast, one in five cirrhotic livers removed at transplant has increased hepatic iron content, with nearly half of those demonstrating iron concentrations comparable to that seen in hereditary hemochromatosis $(\mathrm{HH}){ }^{8}$ Despite the prevalence of these dramatic abnormalities, the pathophysiology of dysregulated iron metabolism in liver disease remains incompletely understood, and the reason that hemosiderosis develops in some patients with chronic liver disease but not others is unknown.

In keeping with the association between hemosiderosis and cirrhosis discussed above, a number of reports have linked iron deposition to more advanced stages of hepatic fibrosis. ${ }^{9-11}$ Some authors have proposed that the relationship between hemosiderosis and advanced fibrosis is causal in nature, i.e. excess iron exacerbates oxidative stress, thereby accelerating hepatic fibrogenesis. ${ }^{12,13}$ Other investigators have concluded that iron deposition is a surrogate marker for advanced fibrosis rather than a profibrogenic factor per se. ${ }^{14}$ To date, it remains unclear whether hemosiderosis is a cause or a consequence of advanced hepatic fibrosis.

The presence of excess iron in cirrhotic livers has also been linked to worsening liver dysfunction. Nearly 6 decades ago, Zimmerman et al. ${ }^{15}$ reported that cirrhotics with hemosiderosis on liver biopsy manifested greater impairment of liver function compared to non-hemosiderotic cirrhotics. That observation was supported by a more recent study that found that cirrhotics with hemosiderosis on index liver biopsy were significantly more likely to be classified as 
Child's class B or C and to have higher model for end-stage liver disease (MELD) scores than cirrhotics without stainable iron on index liver biopsy. ${ }^{16}$ Like the relationship between iron and fibrosis, it is possible on the one hand that excess hepatic iron worsens liver function, while on the other, iron accumulation may be an epiphenomenon of deteriorating liver function, perhaps as a result of reduced production of the liverspecific iron-regulatory hormone hepcidin.

We hypothesized that an examination of temporal changes in iron metabolism and its potential clinical associations might provide insight into mechanisms responsible for secondary iron deposition in chronic liver disease. Thus, the aim of this study was to identify clinical factors associated with the development of hemosiderosis using biopsy findings and clinical data from a group of patients in whom these data were available at two different time points. Our results indicate that the development of hemosiderosis is associated with worsening anemia and alterations in red blood cell indices suggestive of hemolysis.

\section{Methods}

\section{Patients}

Subjects included in this study were consecutive liver transplant recipients 18 years of age or older who had undergone liver biopsy prior to liver transplantation, the latter having been performed between January 1, 2000 and December 31, 2010 at the University of Iowa Hospitals and Clinics. In all cases, biopsies had been performed at the discretion of the treating physician. Exclusion criteria included prior liver transplantation, less than 1 month between time of biopsy and transplant, transplantation performed to address fulminant liver failure or noncirrhotic liver disease, proven homozygous $\mathrm{HH}$, incomplete clinical records and/or pathological material unavailable for review. Medical records were reviewed for demographic data, information regarding cause/s of liver disease and complications of liver disease (bleeding episodes, blood transfusions, placement of a transjugular intrahepatic portosystemic shunt (TIPS), intractable ascites, hepatic encephalopathy, hepatorenal syndrome, and hepatocellular carcinoma). Laboratory data obtained within 1 month of the biopsy and at transplantation were recorded, including complete blood count and red blood cell indices, measures of iron status (serum iron, total iron binding capacity, and serum ferritin), hepatocyte function and damage markers (serum albumin, aspartate aminotransferase, alanine transaminase) and calculated MELD scores and its constituents (international normalized ratio [INR], creatinine, total bilirubin). The institutional review board of the University of Iowa approved this study and waived patient consent.

The disposition of the 295 adult patients identified on the initial search is shown in Fig. 1. Of these, 192 were excluded for the following reasons: no prior biopsy $(n=120)$, prior biopsy performed at an outside hospital $(n=38)$, incomplete clinical records or pathological material unavailable $(n=13)$, less than 1 month between time of biopsy and transplant $(n=$ $11)$, prior transplantation ( $n=5)$, transplantation for noncirrhotic liver disease $(n=3)$, and proven $\mathrm{HH}(n=2)$.

The distribution of hepatocyte iron scores as a function of their change in the interval between biopsy and transplant in the remaining group of 103 subjects is shown in Fig. 2. The figure includes four cases in which the iron scores decreased between the two time points. Because of the small number of such cases, these patients were not included in subsequent analyses, leaving a total of 99 individuals. For the main statistical analysis, the subjects were divided into two groups, namely those whose hepatocyte iron scores increased between liver biopsy and transplant (Group A, $n=39$ ) and those whose iron scores did not change between these time points (Group B, $n=53$ ). Subjects with $4+$ hepatocyte iron on index biopsy that was unchanged at the time of transplant ( $n$ $=7$ ) were not included in this analysis since no increase in iron score was possible in those with the maximum score at baseline; however, these subjects were included in a second analysis as described below.

\section{Histopathology}

Sections from pre-transplant biopsies and explanted livers were stained with hematoxylin and eosin and Perls' Prussian blue using standard methods in the University of Iowa Pathology Department. Iron staining was graded using the system of Scheuer applied to the average appearance of the specimen. ${ }^{17,18}$ Fibrosis was graded according to the Batts and Ludwig staging system for chronic hepatitis. ${ }^{19}$

\section{Statistical methods}

The data were modeled using five different classes of variables. These included the following: categorical level variables for Groups A and B described above and the variable repeat, indicating biopsy [T1] or explant [T2]; categorical variables of gender and ethnicity; categorical Boolean variables, including etiology of chronic liver disease, history of gastrointestinal bleeding, history of transfusion, history of refractory ascites, history of hepatorenal syndrome, hepatocellular carcinoma, TIPS placement; state variables, including interval from biopsy to transplant, age; and measurement variables, including laboratory values and MELD score. The categorical variables gender and ethnicity and the categorical Boolean variables were analyzed using SAS proc freq (SAS Institute, Cary, NC, USA). The chi-square statistics for the effect of group level variable (Group A or B, T1 or T2) were computed. The chi-square statistics were supplemented by versions reflecting likelihood ratio, continuity-adjustment and Mantel-Haenszel odds ratio. SAS proc glm with Welch's ANOVA was used to analyze the state variables (interval from biopsy to transplant, age).

SAS proc mixed was used to analyze all measurement variables. The raw data were formatted to be used in linear mixed modeling with repeated measures. The format used the two-level variables described above, such that there were two records for each subject, one at biopsy (T1) and one at transplant (T2). The state variable timepoint was equal to 1 at $\mathrm{T} 1$ and $\mathrm{N}$ at T2, where $\mathrm{N}$ was measured in days. The fixed effects in the generalized linear model included group (A vs. $B$ ), repeat (T1 vs. T2) and the group by repeat interaction. With two groups (group, i.e. A, B) and two measurements (repeat, i.e. T1, T2), the output included the covariance parameter estimates, fit statistics, least square means and difference of least square means. A $p$-value $<0.05$ was considered significant.

Pearson correlation coefficients were calculated with Python 3.6 using scipy.stats, pandas, seaborn, and matplotlib. A $p$-value $<0.05$ was considered significant. Correlation 
Fierro-Fine A. et al: Hemosiderosis in end-stage liver disease

Adult Liver Transplant Recipients $\mathrm{n}=295$

\begin{tabular}{l}
\multicolumn{1}{c}{192 excluded } \\
No prior biopsy $(n=120)$ \\
Biopsy done elsewhere $(n=38)$ \\
Incomplete records or pathological \\
material unavailable $(n=13)$ \\
Biopsy done $<1$ mo before transplant $(n=11)$ \\
Prior liver transplant $(n=5)$ \\
Liver transplant for noncirrhotic liver \\
disease $(n=3)$ \\
Proven hereditary hemochromatosis $(n=2)$ \\
*excluded from initial analysis \\
*excluded from both analyses
\end{tabular}

\begin{tabular}{|c|}
\hline $\begin{array}{c}\text { Iron score } \\
\text { increased } \\
\text { between bx and } \\
\text { txp } \\
(n=39, \text { Group A })\end{array}$ \\
\hline $\begin{array}{c}4+\text { iron on both } \\
\text { bx and txp }(n= \\
7)^{\star}\end{array}$ \\
\hline
\end{tabular}

103 evaluated

No change in iron score between bx and txp $(n=53$, Group B)

Iron score decreased between bx and txp $(n=4)^{* *}$

Fig. 1. Disposition of transplant recipients considered for inclusion in the study.

A total of 295 adult patients underwent liver transplantation at our institution between January 1, 2000 and December 31, 2010. One hundred and ninety-two patients were excluded for the reasons given. One hundred and three evaluable patients were categorized based on the change in hepatocyte iron scores between pretransplant biopsy and explant as shown.

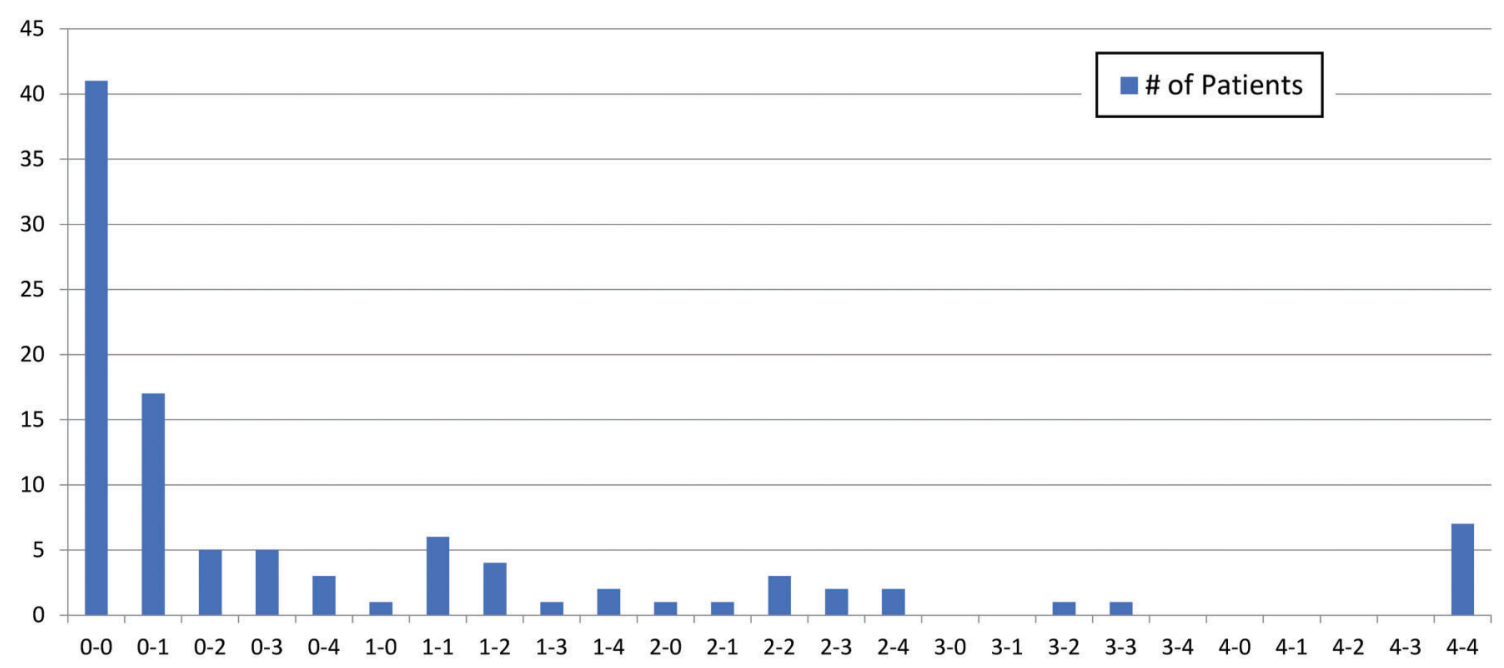

Pretransplant biopsy iron score - explant iron score

Fig. 2. Distribution of changes in iron scores between liver biopsy and liver transplant in 103 subjects.

The first digit in each pair represents the iron score at liver biopsy and the second digit indicates the iron score at liver transplant.

matrix heatmaps were made in RStudio 1.2 .5042 using the ggplot2 and corrplot packages in $\mathrm{R}$ 4.0.0.

\section{Results}

Clinical features of the subjects are given in Table 1. Although the majority of subjects in both Group A (increased hepatocyte iron score between biopsy and transplant) and Group B (no change in iron score) had advanced fibrosis (F3-F4) at biopsy, most had no stainable hepatocyte iron on biopsy and the distribution of iron scores among those with stainable iron was similar between the groups. The groups likewise did not differ significantly with respect to age, sex, ethnicity or etiology of chronic liver disease, history of gastrointestinal bleeding or blood transfusion, intractable ascites, hepatorenal syndrome, hepatocellular carcinoma, and the time interval between biopsy and transplantation (Table 1). Among the categorical variables, only the frequency of TIPS placement was significantly different between Group A (9/39 with TIPS, $23 \%)$ and Group B (4/53 with TIPS, $8 \%)(p=0.03)$.

Hemoglobin concentrations decreased in both groups between biopsy and transplant but the magnitude of the reduction in this parameter was greater in subjects with increased versus stable iron scores in the interval between biopsy and transplant (Group A: $12.6 \pm 0.3 \mathrm{~g} / \mathrm{dL}$ at biopsy, $10.6 \pm 0.3 \mathrm{~g} / \mathrm{dL}$ at transplant; Group B: $12.7 \pm 0.3 \mathrm{~g} / \mathrm{dL}$ at biopsy, $11.4 \pm 0.3 \mathrm{~g} / \mathrm{dL}$ at transplant, $p<0.0001$ for both). Several parameters related to red blood cells differed significantly between the groups. Mean corpuscular hemoglobin $(\mathrm{MCH})$, mean corpuscular hemoglobin concentration (MCHC) and mean corpuscular volume (MCV) tended to increase in both groups between biopsy and transplant, but the 
Fierro-Fine A. et al: Hemosiderosis in end-stage liver disease

\begin{tabular}{|c|c|c|c|}
\hline & $\begin{array}{l}\text { Group A: Increase in iron score } \\
\text { between biopsy and } \\
\text { transplant, } n=39\end{array}$ & $\begin{array}{l}\text { Group B: No change in iron } \\
\text { score between biopsy and } \\
\text { transplant, } n=53\end{array}$ & $p$ \\
\hline Males/females & $28 / 11$ & $43 / 10$ & ns \\
\hline Caucasian/non-Caucasian & $34 / 5$ & $45 / 8$ & ns \\
\hline Age at transplant (years), mean \pm SD & $55.2 \pm 10.4$ & $51.2 \pm 10.7$ & ns \\
\hline $\begin{array}{l}\text { Cause of underlying liver disease: chronic viral } \\
\text { hepatitis/alcoholic disease/non-alcoholic } \\
\text { steatohepatitis/other }^{\text {a }}\end{array}$ & $16 / 14 / 8 / 5$ & $22 / 23 / 6 / 15$ & ns \\
\hline $\begin{array}{l}\text { Interval between biopsy and transplant (days), } \\
\text { mean } \pm \text { SD }\end{array}$ & $907 \pm 927$ & $1,001 \pm 1,036$ & ns \\
\hline Iron score at biopsy $(0 / 1+/ 2+/ 3+)^{\mathrm{b}}$ & $29 / 6 / 4 / 0$ & $42 / 7 / 3 / 1$ & ns \\
\hline Fibrosis stage at biopsy $(1 / 2 / 3 / 4)^{\mathrm{c}}$ & $2 / 1 / 13 / 23$ & $1 / 4 / 14 / 33$ & ns \\
\hline History of GI bleeding & $14(36 \%)$ & $24(45 \%)$ & ns \\
\hline History of blood transfusion & $15(38 \%)$ & $16(30 \%)$ & ns \\
\hline History of intractable ascites & $19(49 \%)$ & $17(32 \%)$ & ns \\
\hline History of hepatorenal syndrome & $10(26 \%)$ & $7(13 \%)$ & ns \\
\hline TIPS placement & $9(23 \%)$ & $4(8 \%)$ & 0.03 \\
\hline History of hepatocellular carcinoma & $7(18 \%)$ & $13(25 \%)$ & ns \\
\hline
\end{tabular}

${ }^{\mathrm{a}}$ All but three cases of chronic viral hepatitis were chronic hepatitis C. There was one case of chronic hepatitis B in each group and 1 HBV-HCV coinfected patient in Group B. Other causes include autoimmune hepatitis, primary biliary cholangitis, primary sclerosing cholangitis. Multiple causes of chronic liver disease were present in some cases.

${ }^{\mathrm{b}}$ Cases with $4+$ iron at biopsy and transplant were not included in this analysis $(n=7)$.

${ }^{\mathrm{C}}$ Information on fibrosis stage missing in one subject.

changes in $\mathrm{MCH}$ and $\mathrm{MCHC}$ were significant only in Group $A$ (Group A MCH: $32.2 \pm 0.5 \mathrm{pg}$ at biopsy, $34.2 \pm 0.5 \mathrm{pg}$ at transplant, $p=0.0002$; Group B MCH: $31.2 \pm 0.4 \mathrm{pg}$ at biopsy versus $32.0 \pm 0.4 \mathrm{pg}$ at transplant, $p=0.05$; Group A MCHC: $33.4 \pm 0.2 \%$ at biopsy, $34.4 \pm 0.2$ at transplant, $p=$ 0.0004 ; Group B MCHC: $33.9 \pm 0.2 \%$ at biopsy, $34.0 \pm 0.2 \%$ at transplant, $p=0.6$ ). The increase in MCV from biopsy to transplant was significant in both groups; however, MCVs were significantly larger in Group A than Group B at both time points ( $96.5 \mathrm{fL}$ in Group A vs. $92.1 \mathrm{fL}$ in Group B at biopsy and $99.2 \mathrm{fL}$ in Group A vs. $94.5 \mathrm{fL}$ in Group B at transplant, $p=0.005$ and $p=0.008$, respectively). Pearson correlations between these parameters and other laboratory variables are shown in Supplementary Fig. 1.

Changes in red blood cell numbers paralleled the changes in hemoglobin, with reductions in red blood cell counts between biopsy and transplant in both groups that were of greater magnitude in Group A (Group A: $4.0 \pm 0.1 \mathrm{M} / \mathrm{mm}^{3}$ at biopsy vs. $3.1 \pm 0.1 \mathrm{M} / \mathrm{mm}^{3}$ at transplant; Group B: $4.2 \pm 0.1$ $\mathrm{M} / \mathrm{mm}^{3}$ at biopsy vs. $3.6 \pm 0.1 \mathrm{M} / \mathrm{mm}^{3}$ at transplant, $p<$ 0.001 for both). Platelet counts fell between biopsy and transplant in both groups, but neither platelet nor white blood cell numbers showed any change in relation to iron scores, suggesting that the observed changes in red blood cell numbers were linked to alterations in iron metabolism in these subjects.

There were significant differences in serum iron studies between the groups at biopsy and at transplant. Even though the prevalence of stainable iron was similar in the groups at biopsy, serum iron levels were higher in Group A compared to Group B at biopsy $(143 \pm 11 \mu \mathrm{g} / \mathrm{dL}$ vs. $99 \pm 9 \mu \mathrm{g} / \mathrm{dL}$, $p=0.002)$ as well as at transplant $(153 \pm 11 \mu \mathrm{g} / \mathrm{dL}$ vs. $84 \pm 11 \mu \mathrm{g} / \mathrm{dL}, p<0.0001)$. Total iron binding capacity
(TIBC) was similar in the two groups at biopsy $(251 \pm 14$ $\mu \mathrm{g} / \mathrm{dL}$ in Group A, $279 \pm 12 \mu \mathrm{g} / \mathrm{dL}$ in Group B, $p=0.14$ ), and was unchanged at transplant in Group $B$ but fell during the interval between biopsy and transplant in Group A (205 \pm $16 \mu \mathrm{g} / \mathrm{dL}$ in Group A vs. $285 \pm 16 \mu \mathrm{g} / \mathrm{dL}$ in Group B at transplant, $p=0.02$ ). As a consequence, saturation of TIBC with iron was significantly higher at biopsy in Group $A$ and rose further in these subjects at transplant, while this parameter was unchanged in Group B (59 $\pm 4 \%$ in Group A vs. $38 \pm 3 \%$ in Group $B$ at biopsy, $p=0.0002 ; 76 \pm 5 \%$ in Group $A$ at transplant, $34 \pm 5 \%$ in Group B at transplant, $p<0.0001$ ). Serum ferritin levels did not differ between the groups at biopsy (Group A: $395 \pm 93 \mathrm{ng} / \mathrm{mL}$ and Group B: $353 \pm 81$ $\mathrm{ng} / \mathrm{mL}, p=0.73$ ) but were significantly higher in Group $A$ versus Group B at transplant $(542 \pm 79 \mathrm{ng} / \mathrm{mL}$ vs. $285 \pm 74$ $\mathrm{ng} / \mathrm{mL}$, respectively, $p=0.02$ ).

MELD scores were significantly higher at transplant in Group A versus Group B, primarily as a result of larger increases in INR and total bilirubin in the former (Group $A$ at transplant $21 \pm 1$ and Group B $15 \pm 1, p=0.004)$. INRs increased over the study interval in both groups but the magnitude of this change was greater in Group A (Group A: $1.4 \pm$ 0.1 at biopsy vs. $2.0 \pm 0.1$ at transplant, $p<0.0001$; Group B: $1.3 \pm 0.1$ at biopsy vs. $1.5 \pm 0.1$ at transplant, $p=0.026$ ). Total bilirubin showed a similar pattern, which was attributable to increases in both direct and indirect fractions (total bilirubin Group A: $2.8 \pm 0.5 \mathrm{mg} / \mathrm{dL}$ at biopsy vs. $7.4 \pm 1.3$ $\mathrm{mg} / \mathrm{dL}$ at transplant, $p=0.0004$; total bilirubin Group B: 2.9 $\pm 0.4 \mathrm{mg} / \mathrm{dL}$ at biopsy vs. $5.3 \pm 1.1 \mathrm{mg} / \mathrm{dL}$ at transplant, $p=0.03)$. Changes in iron scores were not associated with changes in creatinine.

In addition to changes in the magnitude of hepatocyte iron, histopathological examination revealed changes in the 
localization of stainable iron between biopsy and transplant. Nearly $90 \%$ of the livers in Group A exhibited one of the following three patterns: no iron on biopsy with mixed hepatocellular and reticuloendothelial iron on explant; no iron on biopsy with hepatocellular iron on explant; or hepatocellular iron on biopsy, with increased hepatocellular iron on explant. However, there were a few livers that demonstrated other patterns (e.g., mixed or reticuloendothelial iron on biopsy, with hepatocellular iron on explant). Similar variability in the pattern of iron deposition between biopsy and transplant was observed in the approximately one-third of livers in Group B in which some stainable iron was present. Data demonstrating changes in patterns of non-parenchymal cell iron deposition between biopsy and transplant are shown in supplementary Fig. 2. Given the variability of the patterns and the fact that several of the patterns were observed in very small numbers of subjects, the pattern of iron deposition and presence or absence of non-parenchymal cell iron were not analyzed further.

To determine whether there were clinical features that were unique to cirrhotic patients with heavy hepatocellular iron deposition, we repeated the analysis using the same methodology described above but altered the composition of the groups. In this second analysis, the subjects were dichotomized to low-normal iron versus heavy iron deposition groups. The former group comprised subjects with iron scores of 0 and $1+$ at biopsy and at transplant; the latter included those with $3+$ or $4+$ at biopsy and/or transplant. This analysis included the seven subjects with $4+$ iron on biopsy and transplant who were excluded from the initial analysis but excluded subjects with 2+ iron at biopsy and/ or transplant $(n=12)$, as this score does not fit neatly into either the low-normal or heavy iron categories. The heavy iron group had higher baseline liver iron and MELD scores and significantly shorter intervals between biopsy and transplantation, but the laboratory parameters that differed between the low-normal iron and heavy iron groups were otherwise similar to those described above. The data from the second analysis are provided in Supplementary Tables 1-4.

\section{Discussion}

The objective of this study was to identify factors that correlate with development of hemosiderosis in patients with chronic liver disease by comparing semiquantitative assessments of stainable hepatocyte iron in pre-transplant liver biopsies with liver explants from the same individuals. Although most of the subjects in this study had advanced fibrosis at the time of biopsy, the majority had no hepatocellular iron deposition at that point. This observation argues against an important role of iron deposition in driving fibrosis progression, since if that were the case, iron deposition should have preceded the development of advanced fibrosis rather than occurring after advanced fibrosis was already present. On the other hand, the disproportionate worsening of several parameters related to hepatic metabolic function (bilirubin, INR and transferrin [TIBC]) in the subjects whose iron scores increased is consistent with earlier studies linking hemosiderosis to greater impairment of hepatic function. ${ }^{15,16}$

Table 2. Hematologic parameters at liver biopsy and at liver transplant in subjects whose iron scores increased (Group A) versus those whose iron scores remained stable (Group B)

\begin{tabular}{|c|c|c|c|c|}
\hline & Group A, biopsy & Group A, transplant & Group B, biopsy & Group B, transplant \\
\hline Hemoglobin, g/dL & $12.6 \pm 0.3$ & $10.6 \pm 0.3^{b, c}$ & $12.7 \pm 0.3$ & $11.4 \pm 0.3^{a}$ \\
\hline Hematocrit, \% & $37.5 \pm 0.9$ & $30.8 \pm 0.8^{b, c}$ & $37.8 \pm 0.8$ & $33.9 \pm 0.7^{a}$ \\
\hline $\mathrm{MCH}, \mathrm{pg}$ & $32.2 \pm 0.5$ & $34.2 \pm 0.5^{b, c}$ & $31.2 \pm 0.4$ & $32.0 \pm 0.4$ \\
\hline $\mathrm{MCHC}, \%$ & $33.4 \pm 0.2$ & $34.4 \pm 0.2^{b}$ & $33.9 \pm 0.2$ & $34.0 \pm 0.2$ \\
\hline $\mathrm{MCV}, \mathrm{fL}$ & $96.5 \pm 1.2^{a}$ & $99.2 \pm 1.3^{b, c}$ & $92.1 \pm 1.0$ & $94.5 \pm 1.1^{\mathrm{a}}$ \\
\hline $\mathrm{RBC}, \mathrm{M} / \mathrm{mm}^{3}$ & $4.0 \pm 0.1$ & $3.1 \pm 0.1^{b, c}$ & $4.2 \pm 0.1$ & $3.6 \pm 0.1^{a}$ \\
\hline Platelets, $\mathrm{K} / \mathrm{mm}^{3}$ & $110+/-12$ & $78 \pm 9^{b}$ & $115 \pm 10$ & $93 \pm 8^{a}$ \\
\hline $\mathrm{WBC}, \mathrm{K} / \mathrm{mm}^{3}$ & $5.4 \pm 0.4$ & $5.2 \pm 0.4$ & $5.0 \pm 0.3$ & $5.3 \pm 0.4$ \\
\hline
\end{tabular}

${ }^{\mathrm{a}}$ Significant difference compared to Group B at biopsy.

${ }^{\mathrm{b}}$ Significant difference compared to Group A at biopsy.

${ }^{\mathrm{c}}$ Significant difference compared to Group B at transplant.

Table 3. Serum iron parameters at liver biopsy and at liver transplant in subjects whose iron scores increased (Group A) versus those whose iron scores remained stable (Group B)

\begin{tabular}{|c|c|c|c|c|}
\hline & Group A, biopsy & Group A, transplant & Group B, biopsy & Group B, transplant \\
\hline Serum iron, $\mu \mathrm{g} / \mathrm{dL}$ & $143 \pm 11^{a}$ & $153 \pm 11^{c}$ & $99 \pm 9$ & $84 \pm 11$ \\
\hline Total iron binding capacity, $\mu \mathrm{g} / \mathrm{dL}$ & $251 \pm 15$ & $205 \pm 16^{\mathrm{b}, \mathrm{c}}$ & $279 \pm 12$ & $285 \pm 16$ \\
\hline TIBC saturation, \% & $59 \pm 4^{a}$ & $76 \pm 5^{b, c}$ & $38 \pm 3$ & $34 \pm 5$ \\
\hline Ferritin, ng/mL & $395 \pm 93$ & $542 \pm 79^{c}$ & $353 \pm 81$ & $285 \pm 74$ \\
\hline
\end{tabular}

${ }^{a}$ Significant difference compared to Group B at biopsy.

${ }^{b}$ Significant difference compared to Group A at biopsy.

${ }^{\mathrm{c}}$ Significant difference compared to Group B at transplant. 
Fierro-Fine A. et al: Hemosiderosis in end-stage liver disease

Table 4. MELD score, direct and indirect bilirubin at liver biopsy and at liver transplant in subjects whose iron scores increased (Group A) versus those whose iron scores remained stable (Group B)

\begin{tabular}{lllll}
\hline & Group A, biopsy & Group A, transplant & Group B, biopsy & Group B, transplant \\
\hline MELD score & $13 \pm 1$ & $21 \pm 1^{\mathrm{b}, \mathrm{c}}$ & $12 \pm 1$ & $15 \pm 1^{\mathrm{a}}$ \\
INR & $1.4 \pm 0.1^{\mathrm{a}}$ & $2.0 \pm 0.1^{\mathrm{b}, \mathrm{c}}$ & $1.3 \pm 0.1$ & $1.5 \pm 0.1^{\mathrm{a}}$ \\
Total bilirubin, $\mathrm{mg} / \mathrm{dL}$ & $2.8 \pm 0.5$ & $7.4 \pm 1.3^{\mathrm{b}}$ & $2.9 \pm 0.4$ & $5.3 \pm 1.1^{\mathrm{a}}$ \\
Direct bilirubin, $\mathrm{mg} / \mathrm{dL}$ & $1.3 \pm 0.3$ & $3.4 \pm 1.0^{\mathrm{b}}$ & $1.4 \pm 0.3$ & $3.0 \pm 0.8$ \\
Indirect bilirubin, $\mathrm{mg} / \mathrm{dL}$ & $1.5 \pm 0.2$ & $2.4 \pm 0.7$ & $1.4 \pm 0.2$ & $1.9 \pm 0.6$ \\
Creatinine, $\mathrm{mg} / \mathrm{dL}$ & $1.1 \pm 0.1$ & $1.4 \pm 0.2$ & $1.0 \pm 0.1$ & $1.4 \pm 0.1^{\mathrm{a}}$ \\
\hline
\end{tabular}

${ }^{\mathrm{a}}$ Significant difference compared to Group B at biopsy.

${ }^{\mathrm{b}}$ Significant difference compared to Group A at biopsy.

${ }^{\mathrm{c}}$ Significant difference compared to Group B at transplant.

Potential causes of iron deposition in the setting of cirrhosis include multiple blood transfusions, medicinal iron administration, portosystemic shunting, increased iron absorption resulting from hepcidin deficiency, and hemolysis (Fig. 3). Blood transfusions result in predominant Kupffer cell iron deposition. ${ }^{20}$ For this reason and because there was no difference in blood transfusions between Groups A and B, it does not appear that transfusions account for differences in hepatocyte iron deposition in our patients. Data regarding the use, doses and adherence with oral iron were not routinely documented in the records, so it was not possible to accurately quantitate oral iron use. Whether iron supplementation and/or other medications contribute to hemosiderosis in cirrhosis should be addressed in future studies.

The finding of an increased prevalence of patients with TIPS in the group with increased iron scores is intriguing. Literature dating back to the era of portacaval shunt surgery suggested that the creation of a portosystemic shunt was sometimes followed by the development of hemosiderosis. ${ }^{21,22}$ Those observations have received little attention since surgical shunts fell out of favor, hence it remains unclear whether the relationship between shunting and siderosis is causal in nature, and if so, by what mechanism. Assuming that TIPS and hemosiderosis might be linked, we considered the possibility that the likelihood of developing hemosiderosis after TIPS might depend on the length of time that the TIPS was in place. However, there was no difference in the time from TIPS placement to liver transplant in Group A (597 \pm 1207 [standard deviation] days) versus Group B (972 \pm 075 days) (non-significant difference), nor did we observe a consistent pattern of localization of iron in the livers of patients with TIPS from either group (data not shown). It would be worthwhile to investigate this question with a larger number of cases.

Given the association of iron deposition with hepatic synthetic dysfunction, it is tempting to assume that

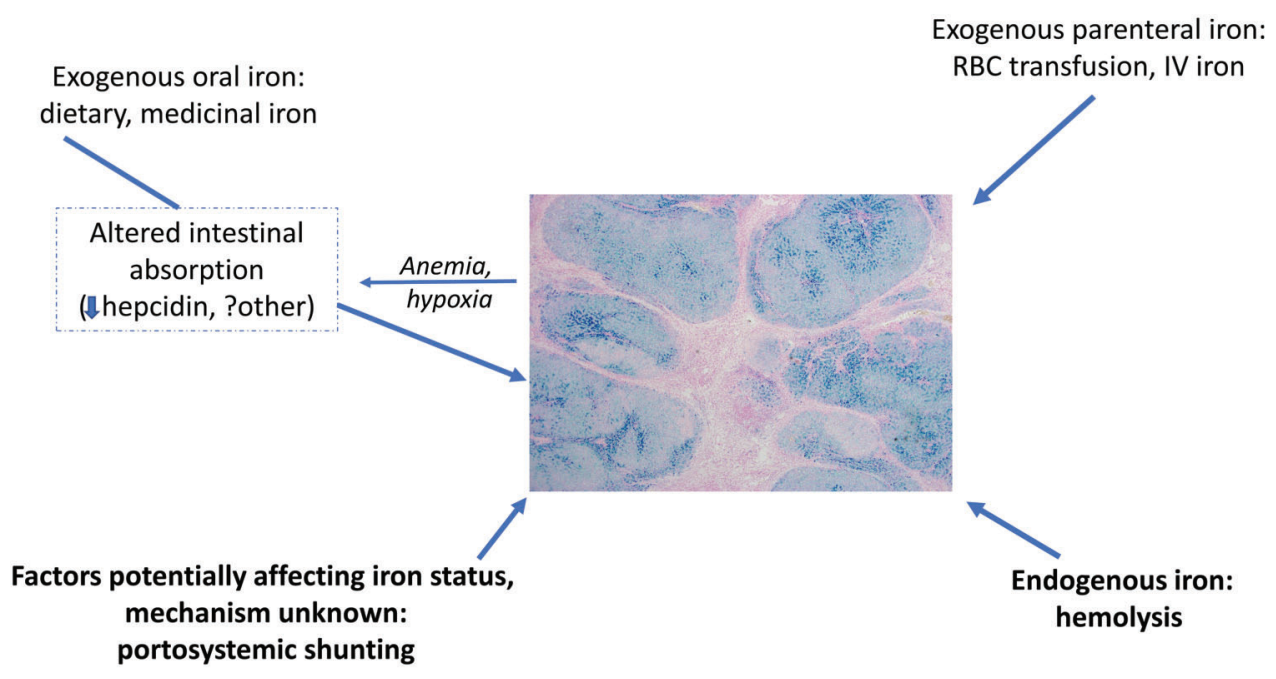

Fig. 3. Potential contributors to hepatocellular iron deposition in cirrhosis.

Uptake of exogenous iron originating from dietary sources and/or medicinal iron from the intestine may be increased due to reduced hepcidin levels. Hepcidin is responsive to a number of physiological stimuli, including anemia and hypoxia, which are potentially relevant in cirrhosis. Transfusion of red blood cells or therapeutic iron infusions are other exogenous sources of iron. An important endogenous source of iron is the hemoglobin of red blood cells, and hemolysis may contribute to iron deposition. Although the mechanism is obscure, portosystemic shunting has also been implicated as a cause of iron loading. Text in bold font indicates processes identified in this study as possibly contributing to hepatocellular iron deposition. Text in italics indicates phenomena known to modulate hepcidin expression. 


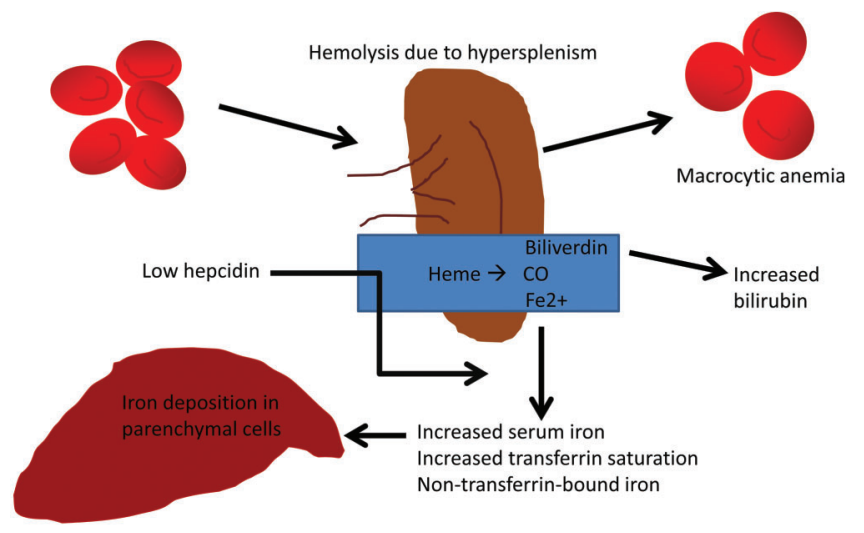

Fig. 4. Hypothetical schema linking extravascular hemolysis to parenchymal iron deposition in cirrhotic livers.

Splenic macrophages catabolize the hemoglobin of red blood cells destroyed in the spleen. Under conditions of low hepcidin, the macrophages release the iron derived from the degraded hemoglobin into the circulation in an unregulated fashion, raising serum iron levels and transferrin saturation. As transferrin becomes saturated with iron, nontransferrin-bound iron (NTBI) appears in the circulation. NTBI is preferentially and efficiently taken up by hepatocytes in a manner that is independent of hepatic iron stores. This sequence of events may explain how iron that is derived from red blood cells destroyed in the spleen contributes to iron loading of liver parenchymal cells.

hemosiderosis is the result of inadequate hepcidin synthesis by a failing liver. A number of studies have addressed the effects of liver disease on hepcidin but the results to date are contradictory. ${ }^{23-26}$ It seems reasonable to assume that decreased hepcidin production may be necessary for hemosiderosis to develop, but whether hepcidin insufficiency is a sufficient cause of parenchymal iron deposition in advanced liver disease is unclear. Note that while liver dysfunction was milder in Group B than Group A, it was still sufficiently severe as to require liver transplantation; yet, the patients in the former group did not demonstrate dysregulated iron metabolism. One possible explanation is that there is a threshold level of liver dysfunction that is required to manifest overt hepcidin deficiency, which was met in Group A but not in Group B. Alternatively, there may be other as-yet unidentified factors that account for the fact that the diseased livers in Group B were able to maintain relatively normal iron homeostasis despite impaired liver function.

An important observation from this study is that subjects who accumulated substantial amounts of hepatocellular iron tended to do so over relatively short time intervals, confirming observations made by Ludwig et al. ${ }^{8}$ The rapid acquisition of liver iron in this context contrasts with the incremental iron accretion that is characteristic of $\mathrm{HH}$, notwithstanding that hemosiderosis and hemochromatosis are both presumed to be caused by hepcidin deficiency. It is possible that hepcidin expression is more profoundly impaired in advanced liver disease than in hemochromatosis, thereby leading to more rapid development of iron overload. A head-to-head comparison of hepcidin levels in these two conditions is needed to determine whether this is the case. Nonetheless, the notion that dysregulated absorption of dietary iron is sufficient to account for rapid development of hemosiderosis in cirrhosis is difficult to reconcile with the realities of anorexia and mal- nutrition that are ubiquitous among patients with end-stage liver disease.

This observation, together with the results of this study, suggest that an endogenous source of iron might contribute to the development of hemosiderosis. Accelerated destruction of red blood cells secondary to hypersplenism in advanced liver disease has been well-described in studies from the 1950's and 60's. ${ }^{27-30}$ Cirrhotics with macrocytic anemia not associated with nutritional deficiencies were shown to have elevated reticulocyte counts, increased numbers of erythrocyte precursors in the bone marrow, and increased urobilinogen excretion. Hemolysis was convincingly demonstrated based on decreased red blood cell survival times, coupled with the finding that transfusion of radiolabeled autologous red blood cells resulted in heavy deposition of radioactivity in the spleen with abnormally little activity in the liver. Furthermore, the half-survival time of transfused red cells correlated with hemoglobin levels in one study, ${ }^{27}$ suggesting that hemolysis contributed significantly to anemia in some cirrhotics.

Findings linking hepatic iron deposition with hemolysis in the present study include the significant worsening of anemia, greater elevation in bilirubin (including the indirect fraction) and more prominent alterations in red blood cell indices between biopsy and transplant in the subjects in Group A. Our observations are in line with recent studies that have linked severity of anemia and macrocytosis with the degree of hepatic dysfunction and portal hypertension in patients with advanced liver disease. ${ }^{31,32}$ Regarding the alterations in red blood cell indices, because evidence for hemolysis was not systematically sought in our subjects, we speculate that the increase in MCV in Group A may be due to increased numbers of circulating reticulocytes, while the higher MCHC might reflect abnormal red blood cell shapes associated with hemolysis (spherocytes or irregularly contracted cells resembling spherocytes). ${ }^{33}$ Alternatively, elevations in $\mathrm{MCH}$ and $\mathrm{MCHC}$ might reflect stimulation of heme and hemoglobin synthesis in erythroid cells driven by high levels of transferrin saturation in Group A. This explanation has been proposed to account for the elevation in $\mathrm{MCH}$ and $\mathrm{MCHC}$ observed in patients with $\mathrm{HH}$, whose hemoglobin levels tend to be modestly increased. ${ }^{34}$ But in contrast to hemochromatosis patients, hemoglobin levels were significantly lower at transplant among the cirrhotic patients with higher levels of transferrin saturation, $\mathrm{MCH}$, and $\mathrm{MCHC}$, which suggests that if hemoglobin synthesis was increased in this group, it was more than counterbalanced by an increase in hemoglobin degradation.

Chronic hemolysis caused by conditions such as thalassemia is well-known to cause hepatic iron overload, but the possibility that extravascular hemolysis secondary to hypersplenism plays a role in the development of hemosiderosis in advanced liver disease has received little attention. Several factors likely account for this. Anemia is common in cirrhosis but comprehensive diagnostic evaluation for the etiology of anemia is overlooked in many of these patients. ${ }^{31}$ In a patient with a known diagnosis of cirrhosis, an elevated bilirubin level may be taken as evidence of worsening liver function, thus no measurement of direct bilirubin is carried out and a potential clue to the presence of hemolysis is missed. Even when hemolysis is considered, interpretation of laboratory tests such as haptoglobin may be confounded by the presence of hepatic synthetic dysfunction. ${ }^{35}$

Another complicating factor is the expectation that iron deposition caused by hemolysis should predominantly affect 
Fierro-Fine A. et al: Hemosiderosis in end-stage liver disease

reticuloendothelial cells. Hemolysis may contribute to the variable amounts of Kupffer cell iron that can be seen in cirrhotic livers but it is not readily evident how hemolysis could account for the preferential hepatocellular iron deposition that is seen in a subset of patients with liver disease. ${ }^{36,37}$ We propose that in the specific context of advanced liver disease with hepcidin deficiency, extravascular hemolysis might give rise to predominant parenchymal cell iron deposition, as outlined in Fig. 4. In this scenario, as red blood cells are destroyed in the spleen, their hemoglobin is catabolized by splenic macrophages. Because of depressed hepcidin levels (due to hepatic failure, hemolysis, ${ }^{38}$ or the combination of these factors), iron derived from degraded hemoglobin is released from macrophages into the circulation in an unregulated fashion, leading to elevated serum iron levels and increased transferrin saturation. (Note that these findings were present in Group A at biopsy and anticipated the subsequent increase in iron scores.) Uptake of transferrin-bound iron is limited by the physiologic mechanisms that regulate transferrin receptor-1 expression, which appear to be intact in cirrhotic human livers, ${ }^{24}$ thus curtailing uptake of iron by this route. As iron continues to be released from splenic macrophages and transferrin becomes saturated with iron, nontransferrin-bound iron appears in the circulation. Non-transferrin-bound iron is preferentially taken up by hepatocytes in an efficient manner that is independent of hepatic iron stores. ${ }^{39,40}$ Coupled with the abnormally low hepatic turnover of radiolabeled red blood cells described above (indicating diminished phagocytosis of erythrocytes by hepatic macrophages), these conditions create the seemingly paradoxical situation in which iron derived from splenic destruction of red blood cells ends up deposited primarily in hepatocytes (Fig. 4).

Although the details of this mechanism are speculative, a similar phenomenon has been shown in patients with alcoholic liver disease complicated by spur cell anemia. Rather than Kupffer cell iron deposition, as would be expected to result from hemolysis and multiple blood transfusions, liver explants from patients with this condition show an $\mathrm{HH}$-like pattern of iron deposition, indicating diversion of iron from macrophages to hepatocytes in this situation. ${ }^{41}$ Along these same lines, we reported that cirrhotics with the $\mathrm{HH}$-like pattern of secondary iron overload tend to have a paucity of splenic iron, suggesting that there is a reciprocal relationship between splenic (macrophage) iron and hepatocyte iron loading. ${ }^{37}$

In summary, our data suggest that extravascular hemolysis caused by hypersplenism may contribute to secondary hepatic iron deposition in cirrhosis. Future studies are needed to confirm these observations. It will be of particular importance to design prospective trials that combine comprehensive evaluation of anemia, detailed information regarding blood loss and administration of supplemental iron as well as dietary iron intake, evaluation of portosystemic shunting and measurements of serum hepcidin with evaluation of intestinal iron absorption in patients with chronic liver disease in order to provide a more comprehensive understanding of the mechanisms that contribute to the development of dysregulated iron metabolism. Other important areas of investigation will be to identify factors that predispose some cirrhotic patients to develop hemolysis, as well as clarifying the nature of the relationship between hemolysis, secondary iron deposition and impairment of liver function.

\section{Acknowledgments}

We thank Innes Hicsasmaz for creating the heatmaps of the correlation matrices.

\section{Funding}

None to declare.

\section{Conflict of interest}

The authors have no conflict of interests related to this publication.

\section{Author contributions}

Study concept and design ( $A F, L G, K E B)$, acquisition of data $(A F, L G)$, analysis and interpretation of data (AF, LG, KEB), statistical analysis $(\mathrm{HH})$, drafting of the manuscript $(A F)$, critical revision of the manuscript (KEB).

\section{References}

[1] Di Bisceglie AM, Axiotis CA, Hoofnagle JH, Bacon BR. Measurements of iron status in patients with chronic hepatitis. Gastroenterology $1992 ; 102: 2108-$ 2113. doi: 10.1016/0016-5085(92)90339-z.

[2] Haque S, Chandra B, Gerber MA, Lok AS. Iron overload in patients with chronic hepatitis C: a clinicopathologic study. Hum Pathol 1996;27:12771281. doi: 10.1016/s0046-8177(96)90337-8.

[3] Piperno A, Vergani A, Malosio I, Parma L, Fossati L, Ricci A, et al. Hepatic iron overload in patients with chronic viral hepatitis: role of HFE gene mutations. Hepatology 1998;28:1105-1109. doi: 10.1002/hep.510280427.

[4] Angulo P, Keach JC, Batts KP, Lindor KD. Independent predictors of liver fibrosis in patients with nonalcoholic steatohepatitis. Hepatology 1999;30: 1356-1362. doi: 10.1002/hep.510300604.

[5] Distante S, Bjøro K, Hellum KB, Myrvang B, Berg JP, Skaug K, et al. Raised serum ferritin predicts non-response to interferon and ribavirin treatment in patients with chronic hepatitis C infection. Liver 2002;22:269-275. doi: 10 . 1046/j.0106-9543.2002.01672.x.

[6] Chitturi S, Weltman M, Farrell GC, McDonald D, Kench J, Liddle C, et al. HFE mutations, hepatic iron, and fibrosis: ethnic-specific association of NASH with C282Y but not with fibrotic severity. Hepatology $2002 ; 36: 142-149$. doi: 10.1053/jhep.2002.33892.

[7] Valenti L, Pulixi EA, Arosio P, Cremonesi L, Biasiotto G, Dongiovanni P, et al. Relative contribution of iron genes, dysmetabolism and hepatitis $C$ virus (HCV) in the pathogenesis of altered iron regulation in HCV chronic hepatitis. Haematologica 2007;92:1037-1042. doi: 10.3324/haematol.11281.

[8] Ludwig J, Hashimoto E, Porayko MK, Moyer TP, Baldus WP. Hemosiderosis in cirrhosis: a study of 447 native livers. Gastroenterology 1997;112:882-888. doi: 10.1053 /gast.1997.v112.pm9041250.

[9] George DK, Goldwurm S, MacDonald GA, Cowley LL, Walker NI, Ward PJ, et al. Increased hepatic iron concentration in nonalcoholic steatohepatitis is associated with increased fibrosis. Gastroenterology 1998;114:311-318. doi: $10.1016 / \mathrm{s} 0016-5085(98) 70482-2$.

[10] Hézode C, Cazeneuve C, Coué O, Roudot-Thoraval F, Lonjon I, Bastie A, et al. Liver iron accumulation in patients with chronic active hepatitis C: prevalence and role of hemochromatosis gene mutations and relationship with hepatic histological lesions. J Hepatol 1999;31:979-984. doi: 10. 1016/s0168-8278(99)80308-0.

[11] Metwally MA, Zein CO, Zein NN. Clinical significance of hepatic iron deposition and serum iron values in patients with chronic hepatitis C infection. Am J Gastroenterol 2004;99:286-291. doi: 10.1111/j.1572-0241.2004.04049.x

[12] Bonkovsky HL, Troy N, McNeal K, Banner BF, Sharma A, Obando J, et al. Iron and HFE or TfR1 mutations as comorbid factors for development and progression of chronic hepatitis C. J Hepatol 2002;37:848-854. doi: 10. 1016/s0168-8278(02)00305-7.

[13] Nelson JE, Bhattacharya R, Lindor KD, Chalasani N, Raaka S, Heathcote EJ, et al. HFE C282Y mutations are associated with advanced hepatic fibrosis in Caucasians with nonalcoholic steatohepatitis. Hepatology 2007;46:723729. doi: 10.1002/hep. 21742.

[14] Guyader D, Thirouard AS, Erdtmann L, Rakba N, Jacquelinet S, Danielou H, et al. Liver iron is a surrogate marker of severe fibrosis in chronic hepatitis C. J Hepatol 2007;46:587-595. doi: 10.1016/j.jhep.2006.09.021. 
[15] Zimmerman $\mathrm{HJ}$, Chomet $\mathrm{B}$, Kulesh $\mathrm{MH}$, McWhorter CA. Hepatic hemosiderin deposits. Incidence in 558 biopsies from patients with and without intrinsic hepatic disease. Arch Intern Med 1961;107:494-503. doi: 10. 1001/archinte.1961.03620040020003.

[16] Kayali Z, Ranguelov R, Mitros F, Shufelt C, Elmi F, Rayhill SC, et al. Hemosiderosis is associated with accelerated decompensation and decreased survival in patients with cirrhosis. Liver Int 2005;25:41-48. doi: $10.1111 / \mathrm{j}$. 1478-3231.2005.01022.x.

[17] Scheuer PJ, Standish RA, Dhillon AP. Scoring of chronic hepatitis. Clin Liver Dis 2002;6:335-347, v-vi. doi: 10.1016/s1089-3261(02)00009-0.

[18] Witte DL, Crosby WH, Edwards CQ, Fairbanks VF, Mitros FA. Practice guideline development task force of the College of American Pathologists. Hereditary hemochromatosis. Clin Chim Acta 1996;245:139-200. doi: 10 . 1016/0009-8981(95)06212-2.

[19] Batts KP, Ludwig J. Chronic hepatitis. An update on terminology and reporting. Am J Surg Pathol 1995;19:1409-1417. doi: 10.1097/00000478199512000-00007.

[20] Batts KP. Iron overload syndromes and the liver. Mod Pathol 2007;20 Suppl 1:S31-S39. doi: 10.1038/modpathol.3800715.

[21] Tisdale WA. Parenchymal siderosis in patients with cirrhosis after portasystemic-shunt surgery. N Engl J Med 1961;265:928-932. doi: 10. 1056/NEJM196111092651903.

[22] Conn HO. Portacaval anastomosis and hepatic hemosiderin deposition: a prospective, controlled investigation. Gastroenterology 1972;62:61-72.

[23] Détivaud L, Nemeth E, Boudjema K, Turlin B, Troadec MB, Leroyer P, et al. Hepcidin levels in humans are correlated with hepatic iron stores, hemoglobin levels, and hepatic function. Blood 2005;106:746-748. doi: 10. 1182/blood-2004-12-4855.

[24] Bergmann OM, Mathahs MM, Broadhurst KA, Weydert JA, Wilkinson N, Howe $\mathrm{JR}$, et al. Altered expression of iron regulatory genes in cirrhotic human livers: clues to the cause of hemosiderosis? Lab Invest 2008;88:13491357. doi: 10.1038/labinvest.2008.95.

[25] Vela D. Low hepcidin in liver fibrosis and cirrhosis; a tale of progressive disorder and a case for a new biochemical marker. Mol Med 2018;24:5. doi: 10. 1186/s10020-018-0008-7.

[26] Varghese J, Varghese James J, Karthikeyan M, Rasalkar K, Raghavan R, Sukumaran A, et al. Iron homeostasis is dysregulated, but the iron-hepcidin axis is functional, in chronic liver disease. J Trace Elem Med Biol 2020;58: 126442. doi: $10.1016 /$ j.jtemb.2019.126442.

[27] Jandl JH. The anemia of liver disease: observations on its mechanism. J Clin Invest 1955;34:390-404. doi: 10.1172/JCI103087.

[28] Jones PN, Weinstein IM, Ettinger RH, Capps RB. Decreased red cell survival associated with liver disease; use of radioactive sodium chromate in measurement of red cell survival. AMA Arch Intern Med 1955;95:93-102. doi: 10 1001/archinte.1955.00250070109013.
[29] Allen FA, Carr MH, Klotz AP. Decreased red blood cell-survival time in patients with portal cirrhosis; correlation of laboratory and clinical findings. J Am Med Assoc 1957;164:955-999. doi: 10.1001/jama.1957.02980090009003.

[30] Hall CA. Erythrocyte dynamics in liver disease. Am J Med 1960;28:541-549. doi: 10.1016/0002-9343(60)90148-0.

[31] Scheiner B, Semmler G, Maurer F, Schwabl P, Bucsics TA, Paternostro R, et al. Prevalence of and risk factors for anaemia in patients with advanced chronic liver disease. Liver Int 2020;40:194-204. doi: 10.1111/liv.14229.

[32] Yang J, Yan B, Yang L, Li H, Fan Y, Zhu F, et al. Macrocytic anemia is associated with the severity of liver impairment in patients with hepatitis $B$ virusrelated decompensated cirrhosis: a retrospective cross-sectional study. BMC Gastroenterol 2018;18:161. doi: 10.1186/s12876-018-0893-9.

[33] Bain BJ. The spherocytic haemolytic anaemias. Br J Haematol 2001;115: 229-230. doi: 10.1046/j.1365-2141.2001.115_1cr-2.x.

[34] Barton JC, Bertoli LF, Rothenberg BE. Peripheral blood erythrocyte parameters in hemochromatosis: evidence for increased erythrocyte hemoglobin content. J Lab Clin Med 2000;135:96-104. doi: 10.1016/s0022-2143(00) 70026-6.

[35] Körmöczi GF, Säemann MD, Buchta C, Peck-Radosavljevic M, Mayr WR, Schwartz DW, et al. Influence of clinical factors on the haemolysis marker haptoglobin. Eur J Clin Invest 2006;36:202-209. doi: 10.1111/j.13652362.2006.01617.x.

[36] Brunt EM, Olynyk JK, Britton RS, Janney CG, Di Bisceglie AM, Bacon BR. Histological evaluation of iron in liver biopsies: relationship to HFE mutations. Am J Gastroenterol 2000;95:1788-1793. doi: 10.1111/j.15720241.2000.02175.x

[37] Abu Rajab M, Guerin L, Lee P, Brown KE. Iron overload secondary to cirrhosis: a mimic of hereditary haemochromatosis? Histopathology 2014;65: 561-569. doi: 10.1111/his.12417.

[38] Nicolas G, Chauvet C, Viatte L, Danan JL, Bigard X, Devaux I, et al. The gene encoding the iron regulatory peptide hepcidin is regulated by anemia, hypoxia, and inflammation. J Clin Invest 2002;110:1037-1044. doi: 10 . 1172/JCI15686.

[39] Brissot P, Wright TL, Ma WL, Weisiger RA. Efficient clearance of nontransferrin-bound iron by rat liver. Implications for hepatic iron loading in iron overload states. J Clin Invest 1985;76:1463-1470. doi: 10. $1172 /$ JCI112125.

[40] Wright TL, Brissot $P, M a$ WL, Weisiger RA. Characterization of non-transferrin-bound iron clearance by rat liver. J Biol Chem 1986;261:10909-10914.

[41] Pascoe A, Kerlin P, Steadman C, Clouston A, Jones D, Powell L, et al. Spur cell anaemia and hepatic iron stores in patients with alcoholic liver disease undergoing orthotopic liver transplantation. Gut 1999;45:301-305. doi: 10.1136/gut.45.2.301. 